\title{
Evaluation of the effect of the interleukin-25 serum concentration on the intensity of the symptoms of atopic dermatitis and epidermal barrier
}

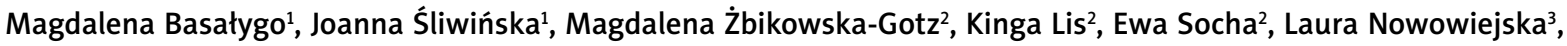 \\ Zbigniew Bartuzi², Barbara Zegarska ${ }^{1}$
}

\begin{abstract}
'Department of Cosmetology and Aesthetic Dermatology, Ludwik Rydygier Collegium Medicum in Bydgoszcz, Nicolaus Copernicus University in Torun, Poland

${ }^{2}$ Department and Clinic of Allergology, Clinical Immunology and Internal Diseases, Ludwik Rydygier Collegium Medicum in Bydgoszcz, Nicolaus Copernicus University in Torun, Poland

3Department of Dermatology and Venereology, Faculty of Medicine, Collegium Medicum in Bydgoszcz, Nicolaus Copernicus University in Torun, Poland
\end{abstract}

Adv Dermatol Allergol 2021; XXXVIII (6): 1071-1077 DOI: https://doi.org/10.5114/ada.2021.109686

\begin{abstract}
Introduction: Interleukin 25 (IL-25) is a cytokine with proinflammatory and anti-inflammatory effects, and its biological function of reciprocal epidermal hyperplasia and of inhibiting the filaggrin synthesis points to an essential role connecting the inflammatory process with damage to the epidermal barrier in the course of atopic dermatitis (AD). Aim: To assess the IL-25 in serum concentration in AD patients and to analyse its possible correlation with the disease intensity and selected epidermal barrier parameters such as transepidermal water loss (TEWL).

Material and methods: The study involved 43 patients with AD and 22 healthy volunteers. The IL- 25 concentration was measured using the ELISA method. The intensity of disease symptoms was investigated using W-AZS and EASI indicators. The epidermal barrier was evaluated using a Tewameter TM300 and Corneometer CM825.

Results: The concentration of IL-25 in serum was higher in the study group than in the control group. IL-25 serum concentration correlates with W-AZS/EASI in patients with a severe and moderate course of AD. The concentration of IL-25 affects the TEWL within the affected, evaluated skin surface.

Conclusions: An elevated IL-25 concentration in serum is characteristic for patients with moderate and severe AD intensity. The IL-25 concentration in serum correlates with TEWL and with the moisture level in the affected area. However, further studies are necessary to determine the role played by IL-25 in the course of the disease and how it affects the functional parameters of the epidermal barrier.
\end{abstract}

Key words: atopic dermatitis, interleukin 25 , transepidermal water loss.

\section{Introduction}

Atopic dermatitis (AD) is a chronic and recurrent dermatosis, clinically defined as dermatitis [1] showing a high intersubject and intrasubject variability [2], affecting more than 230 million people globally [3]. The morphology of the changes includes erythema, itch, dry skin, lichenoid changes, skin plaques, or prurigo nodularis [4]. The key role in $A D$ pathogenesis is attributed to genetic and environmental factors [5]; however, the latest scientific reports focus on immune dysregulation and epidermal barrier disorders [6-8]. An increased epidermal per- meability leads to irritant contact dermatitis [9], which triggers an increased sensitivity to allergens [10] and, as a result, a Th-2 dependent response [11]. Ninety percent of the barrier permeability is due to the stratum corneum 10-20 $\mu \mathrm{m}$ thick [12]. Corneocytes and intercellular lipids (ceramides, cholesterol, free fatty acids) maintaining the integrity of epidermis come from the final phase (of keratinocytes differentiation $[13,14]$ interleukin-25 (IL-25) participates in [15]).

IL-25 is a cytokine that represents the IL-17 family and which shows a unique structure and function [16]. The mass of protein molecule (IL-25) is $16.7 \mathrm{kDa}$ [17], and its

Address for correspondence: Magdalena Basałygo, Department of Cosmetology and Aesthetic Dermatology, Ludwik Rydygier Collegium Medicum, Nicolaus Copernicus University, 13-15 Jagiellońska St, 85-067 Bydgoszcz, Poland, phone: +48 692208123, e-mail: magdalena_basalygo@poczta.onet.pl Received: 25.05.2021, accepted: 22.06.2021. 
structure covers 11 cysteine residues facilitating the formation of disulphide bridges stabilizing specific spaces of the polypeptide chain fragment positioning [18]. As for the function, it is characterised by inducing a Th2-dependent response [19], unlike the other IL-17 family cytokines [20]. By producing Th2 cytokines, IL-25 can induce the production of eosinophiles and IgE [17]. On the other hand, it is attributed with a direct effect on the release of cytokines with a local proinflammatory effect from dendritic cells, macrophages, and from the cells of perivascular parenchyma [21].

In the course of $A D, I L-25$ has an essential inhibiting effect on the final differentiation of keratinocytes: epidermal reciprocal hyperplasia [15]. As a result, it increases transepidermal water loss (TEWL) and leads to a release of the cascade of proinflammatory cytokines [22], including IL-25 [23]. The participation of IL-25 in epidermal barrier disorders also involves reducing the synthesis of filaggrin [24], and thus directly disturbs the protective functions of the skin in atopic patients. A decreased epidermal cohesion, on the other hand, increases the penetration of exogenous allergens, and so it is suggested that IL-25 is a link connecting the inflammation process with epidermal barrier damage [25]. Filaggrin, as the source of free amino acids with a high hygroscopy, is of key importance in TEWL control and the level of stratum corneum moisture [25], and its participation in the AD pathogenesis has already been confirmed in numerous reports [26-28].

To recapitulate, IL-25 takes part in the process of dermatitis, it affects the integrity of the epidermal barrier, and a detailed determination of its role in the course of $A D$ can provide therapeutic benefits [29]. Explaining the factors responsible for the recruitment of inflammatory cells to tissues can be essential for understanding the mechanisms of the occurrence of skin changes in the course of the disease and for developing new diagnostic and therapeutic methods. The reports justify further research on the role of IL-25 in the skin of patients AD [30].

\section{Aim}

The aim of the paper was to assess the effect of IL-25 on the course of $A D$ by: 1) measuring the concentration of IL-25 in serum in the group of patients with $A D$ and in the control group; 2) analysis of the correlation between IL-25 concentrations in serum and the intensity of the disease evaluated following W-AZS and EASI; 3) analysis of the correlation between the measurements of IL-25 concentrations in serum with epidermal barrier parameters: TEWL and epidermal moisture levels.

\section{Material and methods}

The study group consisted of 43 patients with $A D$ aged 18 to 35 years. The average age was 26 years. In the studied patient population, women accounted for $46.51 \%$ (20 persons) and men - for $53.49 \%$ of the subjects (23 persons). The control group, adequately selected in terms of sex and age, consisted of 22 healthy volunteers (12 women and 10 men) aged 22 to 38 years (average age 29 years) denying any symptoms of dermatology and allergy diseases in their personal and family history. Food allergies were diagnosed in $28 \%$ of cases and the sensitization to airborne allergens was recorded in $36 \%$ of the patients with AD. Furthermore, $19 \%$ suffered from bronchial asthma, and rhinitis was observed in 44\% (specifically, non-allergic rhinitis in $27 \%$ and allergic rhinitis in 17\% of the group). Recorded answers concerning the possible hypersensitivity reactions were taken as reflecting the patient's history and were not based on results of diagnostic procedures such as specific IgE, skin prick tests, or oral food challenge tests. AD was diagnosed following the Hanifin and Rajka criteria. The research exclusive criteria for both groups were written consent to participate in the study and being at least 18 years old. The patients included in the study were not allowed to be generally treated for at least a month before the study. The study excluded patients with AD undergoing phototherapy, ciclosporin A therapy, oral and topical corticoids therapy (up to 6 weeks after the end of the therapy), treated with topical preparations being inhibitors of calcineurin: pimecrolimus and tacrolimus, patients undergoing or patients who had completed a specific immunotherapy, breast-feeding and pregnant women, and patients with inflammatory diseases, infections, autoimmune diseases, or with neoplasms. The exclusion criterion included also the application of emollients on the examination date as well as nevi, scars, and injuries in the area. The concentration of IL-25 in serum with the enzyme-linked immunosorbent assay (ELISA) was evaluated using ready-made Human Platinum ELISA sets (eBioscience). The serum was portioned out into Eppendorf-type test tubes and frozen at the temperature of $-82^{\circ} \mathrm{C}$ until assays were made, compliant with the guidelines provided by the set manufacturer.

The severity of skin lesions in AD patients was evaluated based on the Eczema Area and Severity Index (EASI). The EASI scoring system involved an assessment of the skin inflammatory condition intensity divided into 4 areas: the head/neck, trunk, and upper and lower limbs. Each of the above areas was exposed to a 4-point grading (0 to 3), each time considering 4 types of eruptions: erythema, lesion/papules, erosions/neurotic excoriations, and lichenification. The eruptions were correlated with the entire body surface expressed using a 7-point scale. The maximum result measured with EASI is a score of 72. The other scoring system was the W-AZS, which evaluated the subjective symptoms, especially itching and sleep disorders as well as objective symptoms, in particular the extent and intensity of skin changes in all 
the possible locations. The W-AZS evaluated sleep disorders (score 0-12), skin itching (score 0-22), inflammation (score 0-3), and the extent of skin changes (score 0-3). The maximum score according to the $\mathrm{W}$-AZS is 178 .

The TEWL measurement was taken using a Tewameter TM 300, connected to a Cutometr MPA 58 adapter manufactured by Courage-Khazaka Electronics (Köln, Germany), applying standard procedures [31]. All the measurements were taken at a room temperature of $20-22^{\circ} \mathrm{C}$ and $40-60 \%$ relative humidity, permanently controlled with an RHT 100 environment conditions sensor. To guarantee the measurement stability, a Probe Heater PR 100 was used, which maintained the probe at a temperature close to the skin temperature. In the group with $A D$ the examination was made in the affected areas, $2 \mathrm{~cm}$ away from the inflammatory changes, and on seemingly healthy skin - most frequently half the length of the forearm on the internal side. In the control group the area under study included only the forearm on the internal part. To ensure the accuracy of the measurement reading, all the parameters were read at the same time of the day and under fixed light conditions, and $20 \mathrm{~min}$ prior to the examination the patients were allowed some rest in the examination room. Correspondingly, the measurement of epidermal moisture was assayed using a Corneometer CM 825. The data acquired from the TEWL and from the epidermal moisture measurements were recorded using MPA software.

\section{Statistical analysis}

The normality of distributions was verified with the Shapiro-Wilk test. Due to the fact that the assumptions on the use of parametrical methods were not met, the hypotheses were verified with non-parametrical methods. The significance of differences between the groups was verified with the $U$ Mann-Whitney test, and the analysis of correlation was made with Spearman's rank correlation coefficient. The level of significance was assumed at $\alpha=0.05$; the value of the calculated probability meeting the assumption $p<\alpha$ was considered significant. The calculations were made in Statistica 10.0 software from Statsoft Polska.

\section{Results}

The clinical condition of the patients measured with the W-AZS was, on average, $53 \pm 33$ (min. score 7, max. score 114), and according to EASI - on average $18 \pm 15$ (min. score 1; max. score 67). The W-AZS median was 67, while the EASI median was observed as 24 . A comparison of the results of 2 independent disease symptoms intensity indices is shown in Table 1.

In the AD group the average TEWL value within the affected skin was $41.5 \pm 15.7$ [g/m²/h] (min.12.6; max. 82.2). The TEWL values found within the norm (0-25 g/ $\mathrm{m}^{2} / \mathrm{h}$ ) were observed in 6 subjects, whereas TEWL values $>25 \mathrm{~g} / \mathrm{m}^{2} / \mathrm{h}$ pointing to epidermal barrier damage were recorded in 37 (86\%) patients. The TEWL value $2 \mathrm{~cm}$ away from the affected area was on average $37 \pm 15 \mathrm{~g} /$ $\mathrm{m}^{2} / \mathrm{h}$ ( $\min .10$; max. 75), whereas within the seemingly healthy skin it was $18.9 \pm 6.9 \mathrm{~g} / \mathrm{m}^{2} / \mathrm{h}$ (min. 8; max. 30). The control group noted the average TEWL value equal to $13.7 \pm 5.1 \mathrm{~g} / \mathrm{m}^{2} / \mathrm{h}$ (min. 4.1; $\left.\max .24 .6\right)$, and all the values in that group were found within the norm $\left(<25 \mathrm{~g} / \mathrm{m}^{2} / \mathrm{h}\right)$. In the study group the mean measurement of epidermal moisture was 18.8 arbitrarily assumed units \pm 7.1 for the affected skin (min. 3.8; max. 30.0), $24.3 \pm 5.82 \mathrm{~cm}$ away from the affected area (min. 13.2; max. 36.2), and 29.4 \pm 5.2 for seemingly healthy skin (min. 18.9; max. 49.6). The hydration in the stratum corneum in the control group was $48.6 \pm 5.6$ ( $\min$. 40.1; max. 58.2), and the results fell within the norm. The results are presented in Table 1.

The mean values of the concentration of IL-25 pq/ml in serum were higher in the patients with $A D$ (Figure 1) and they were, on average, $145 \pm 98 \mathrm{pq} / \mathrm{ml}$ in the study group and $74 \pm 23 \mathrm{pq} / \mathrm{ml}$ in the control group. The differences were observed to be significant (U Mann-Whitney test: $U=208.5 ; p=0.004$ ).

Table 1. The severity and extent of skin lesions in patients with atopic dermatitis (AD), epidermal barrier parameters patients with $A D$ vs. control subjects

\begin{tabular}{lcc}
\hline Variable & Atopic dermatitis $(n=43)$ & Control group $(n=22)$ \\
\hline W-AZS & $52.63 \pm 32.62$ & - \\
\hline EASI & $17.96 \pm 15.36$ & - \\
\hline TEWL in the lesional skin area & $41.48 \pm 15.71$ & - \\
\hline TEWL at a distance of 2 cm from the lesion of AD & $37.07 \pm 15.15$ & $13.7 \pm 5.12$ \\
\hline TEWL seemingly healthy skin & $18.91 \pm 6.9$ & - \\
\hline Skin hydration level in the lesional skin & $18.76 \pm 7.06$ & - \\
\hline Skin's hydration level 2 cm from the lesion of AD & $24.28 \pm 5.78$ & $29.44 \pm 5.22$ \\
\hline Skin hydration level seemingly healthy & $48.57 \pm 5.57$ & \\
\hline$n-$ number of respondents, $W$-AZS - Index for Atopic Dermatitis, EASI - Eczema Area and Severity Index.
\end{tabular}



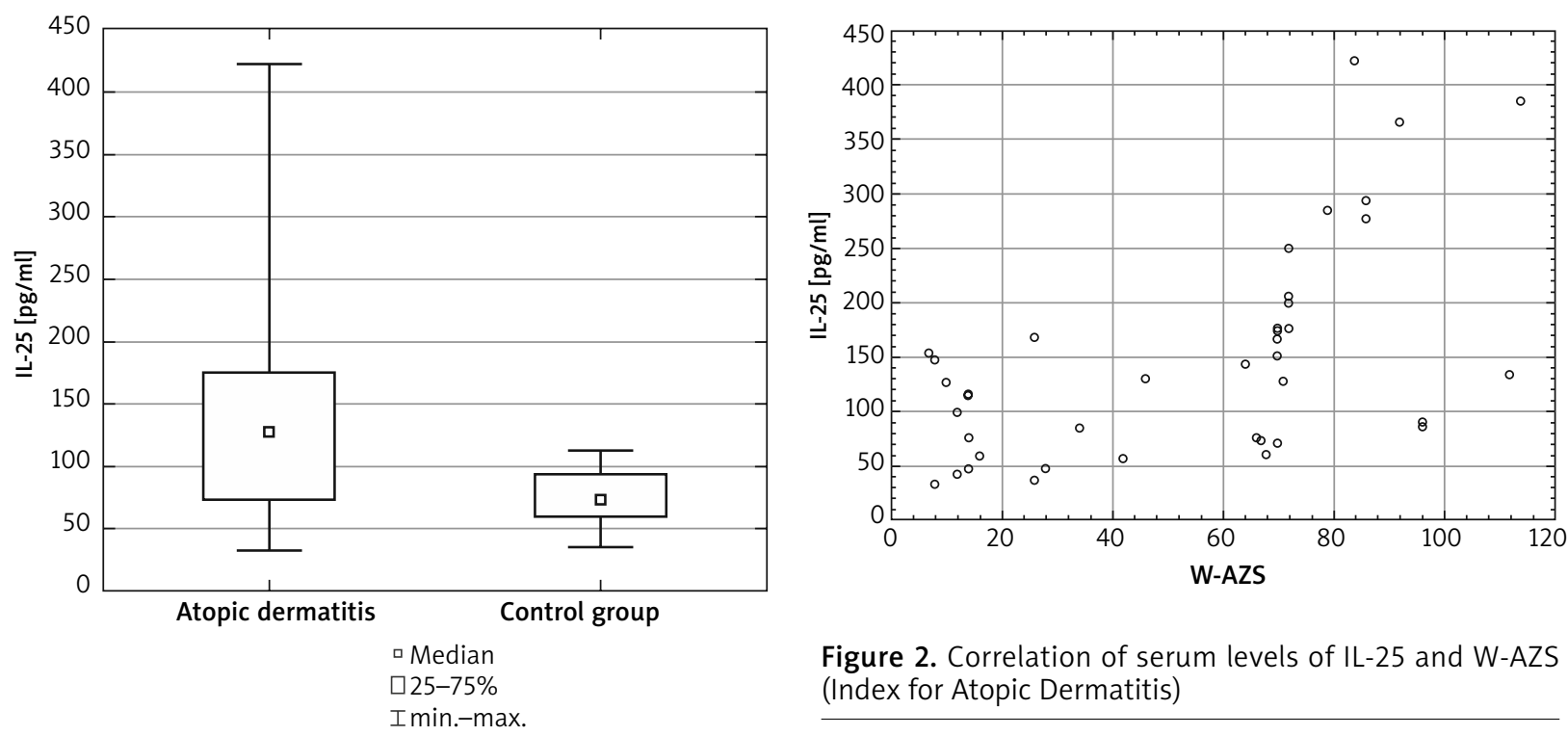

Figure 2. Correlation of serum levels of IL-25 and W-AZS (Index for Atopic Dermatitis)

Figure 1. Comparison of IL-25 concentration

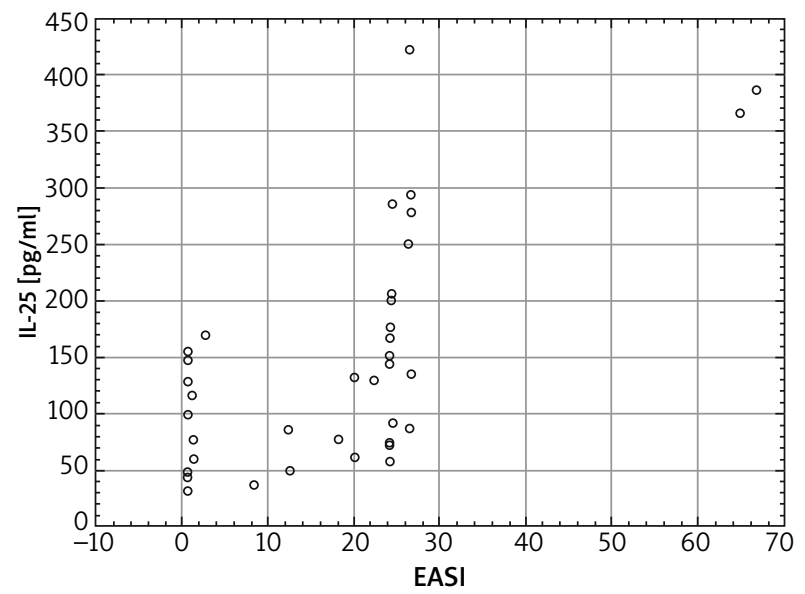

Figure 3. Correlation of serum levels of IL-25 and EASI (Eczema Area and Severity Index)

The verification of the significance of changes in the concentration of $\mathrm{IL}-25 \mathrm{pq} / \mathrm{ml}$ in serum in terms of the intensity of skin changes (W-AZS Index) demonstrated a high positive correlation; Spearman's rank correlation coefficient was 0.61 at $p<0.001$. The data are presented in Figure 2. Because the scatter diagram presented in Figure 2 suggests that up to the score of 60 in the W-AZS index no tendency towards an increase in the concentration of IL-25 in serum was observed, a separate analysis of variance was made in the range below and above the W-AZS score of 60 . The Spearman's rank correlation confirmed the assumptions that in the W-AZS score range below 60 the results of the concentration of IL-25 in serum are not significantly correlated with the in-

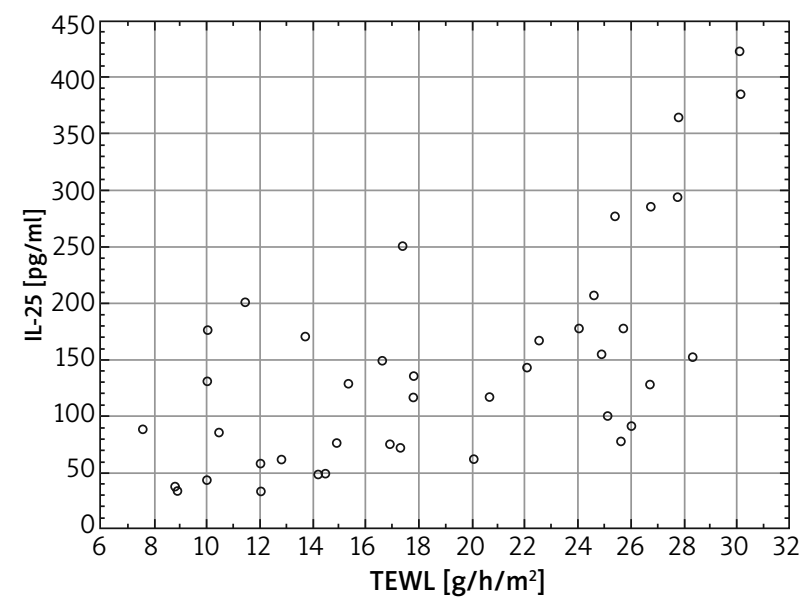

Figure 4. Correlation of serum levels of IL-25 and TEWL seemingly healthy skin

tensity of skin lesions (coefficient of correlation $R=0.01$; $p=0.961$ ), while once that W-AZS value is exceeded, the correlation is significant $(R=0.52 ; p=0.012)$. It points to the correlation with a clinical activity of the disease only in patients with a severe and moderate course of the disease.

Similar correlations were noted for EASI; Spearman's rank correlation coefficient $R=0.65$ points to the positive correlation with the concentration of IL-25 in serum at a significant level $(p<0.001)$. Interestingly, however, the EASI scoring shows a " 0 " value in 11 patients with a high variation in the concentration of IL-25 in serum (Figure 3). A growing EASI value for an elevated concentration of IL-25 is, however, maintained, which supports a correlation. 
There were also evaluated changes in the epidermal barrier parameters depending on the concentration of IL-25 in serum. The strongest correlations were recorded for the affected skin for the TEWL measurement; Spearman's rank correlation coefficient value was $0.74(p<0.001)$, which demonstrates a strong significant correlation between the TEWL in the affected areas and the concentration of IL-25 in serum (Figure 4). For the TEWL measurement, the correlation was also significant for the skin $2 \mathrm{~cm}$ away from inflammatory changes $(R=0.71, p<0.005)$, as well as on the apparently healthy skin $(R=0.62, p<0.005)$. In all the 3 locations a higher IL-25 level in serum correlated with higher TEWL values. Whereas, as for the epidermal moisture level, a negative correlation was observed; Spearman's rank correlation coefficient value was $R=-0.63$ $(p<0.005)$ for the affected skin and $R=-0.61(p=0.0049)$ $2 \mathrm{~cm}$ away from the affected areas. For the seemingly healthy skin, there no significant correlation was noted $(R=-0.58, p>0.005)$ (Figure 5).

\section{Discussion}

Even though barrier function disorders and immune disorders are well known in atopic dermatitis, recently has it become clear that the 2 factors regulate each other and are correlated [6]. According to the latest reports, cytokines Th2 [32] and recently also IL-25 [24, 33] regulate important epidermal barrier functions, especially callused skin and AMP production, as well as inhibiting the major basic proteins of the final differentiation of keratinocytes, especially filaggrin, loricrin, and involucrin [32]. Based on those observations, the aim of the paper was to evaluate the effect of IL-25 on the course of atopic dermatitis, considering the visual intensification of the disease symptoms with 2 independent scales and the functional parameters of the epidermal barrier.

This study demonstrated that the concentration of IL-25 in serum was higher in patients with $A D$ than in the control group. The elevated concentration of IL-25 was recorded also in genetic studies, both in mouse models with $A D$ and in patients with $A D$ [34]. Immunohistochemistry studies have also demonstrated increased IL-25 expression in atopic-dermatitis affected skin, as compared with skin without any disease changes [24]. The allergen provocation test also confirmed an increased IL-25 expression and its receptor in the dermis in $A D 72 \mathrm{~h}$ after a subcutaneous allergen injection [35], which suggests the role of IL-25 at a late stage of $A D$ [36]. However, Aalberse et al. noted a very high IL-25 concentration (above 13,000 pq/ml) in serum after provocation in children allergic to peanuts, which facilitated the conclusion that an increased IL-25 level can be a symptom of a severe atopic phenotype [37]. Totally different observations were made by Martel et al.; studying the culture of cells in patients with $A D$ with flow cytometry, they did not document the pres-

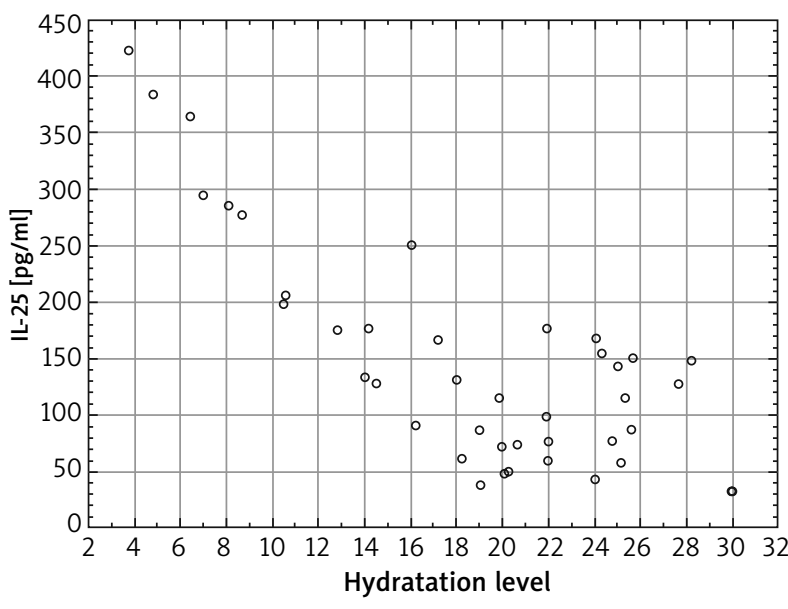

Figure 5. Correlation of serum levels of IL-25 and skin's hydratation level in the lesional skin

ence of IL-25 [38], irrespective of the AD type (endogenous/exogenous). As the cause the authors indicate a low count of cells in the culture. The result of the research can also be due to a low value of EASI scoring; the value did not exceed the score of 10 in anyone, except for 1 patient, which can point to a mild form of atopic dermatitis. The above reports prove that in the course of AD an elevated concentration of IL-25 as compared with the control group is observed. However, it can depend on the disease intensity. Applying various research methodologies does not allow a final conclusion to be drawn.

Further analysis of our own research has demonstrated correlation between the concentration of IL-25 in serum and the intensity of skin lesions measured with the quality scale. However, the correlation for W-AZS was visible only in patients in a moderate/severe condition. Nakamura et al. proved correlation between the concentration of IL-25 and the visual 5-degree scale of skin dryness and redness, as well as the NRS itching scale [39], which, at the same time, points to the participation of IL-25 in epidermal barrier damage. However, there was not demonstrated only the relationship with the symptoms of lichenification, which can show that IL-25 can play a role in the pathogenesis of $A D$ changes both in acute and chronic atopic dermatitis. The lack of high correlation between the concentrations of interleukins in serum and skin changes is, by other authors [40], attributed the profile of cycling cytokines reflecting an activation of $T$ lymphocytes and other cells that we know to produce cytokines, especially eosinophils, basophils, macrophages, and cell responses outside the skin [41]. Hamilton et al., on the other hand, claim that in $A D$ it is difficult to determine quantitatively the erythema and lichenification due to the lack of differentiation between the affected and unaffected areas, as compared with psoriasis [42]. The results of our own research and the research cited 
suggest that IL-25 can be a marker differentiating the severity of the course of atopic dermatitis, especially in patients with a severe and moderate course of the disease.

The present research proved the dependency of the concentration of IL-25 in serum on the epidermal barrier parameters. Similar observations were made by Nakamura et al. [39] with tape-stripping to assess IL-25 in affected skin. Even though the study involved other areas: the cheek, cubital fossa, and the trunk, a weak yet significant correlation was found between SCIL-25 expression and TEWL. Weaker correlations between IL-25 and TEWL, as compared with our own study, and a lack of correlation with the level of hydration of the stratum corneum can also be a result of a smaller inflammatory condition due to treatments with calcineurin inhibitors, local glycocorticosteroids, and antihistamines administered to those patients. Also, the treatment with histamine receptor antagonist could have helped repair the barrier [43]. The argument for the effect of IL-25 on the epidermal barrier includes a well-documented effect of IL-25 on lowering the expression of profilaggrin in the study of the monolayer culture [24], which shows that the cytokine can play a role in the differentiating and functioning of the epidermis [30], leading to an increase in the TEWL value.

As for the unaffected skin, the present study recorded a correlation between the concentration of IL-25 and TEWL. Even though it was previously suggested that the seemingly healthy skin in people with AD shows functional parameter disfunctions and immune disorders [44, 45], the biomarkers not showing any relationship with the visual evaluation of the symptoms intensity were not considered credible. Therefore Ungar et al. indicate that a disease severity evaluation model must be developed which would consider both the activation of cytokines in peripheral blood and the evaluation of seemingly healthy skin [41]. Referring back to the opinion of the researcher, the evaluation of the functional parameters of the skin (TEWL/stratum corneum moisture measurement) in 3 locations seems a good solution as AD changes frequently do not give clinical disease symptoms despite the functional parameter skin disorders [46]. Drawing on our own study results and on the reports of other authors, we can suppose that an increase in the concentration of IL-25 leads to an increase in TEWL in the affected skin.

The research performed, however, shows some limitations. The limitation is an excessively small study group and not specifying the concentration of IgE in serum. Due to the sample size, it was not possible to make an indepth study of the differences between the serum profiles in patients with exogenous and endogenous AD. It is suggested that the effect of a single biomarker cannot be accounted for with a single mechanism, and each AD endotype shows a different unique biological mechanism [47]. The results, however, can be considered explorative data which sketch potential trends and which can provide a springboard for further research.

\section{Conclusions}

Because the participation of IL-25 in AD pathogenesis and the epidermal barrier damage mechanism are not fully known, further research in this field is required. The research results cited and our own observations demonstrate that in AD patients the concentration of IL-25 is significantly higher; however, the relationship with clinical disease symptoms is characteristic only for patients with a moderate and severe course of the disease. The value of the concentration of IL-25 in serum is higher in patients with a higher TEWL value and lower hydration value in the stratum corneum in the affected area. Further research is required to specify the role of IL-25 in the severity of the course of the disease and to demonstrate how it affects the TEWL value and the epidermal moisture level, both in affected skin and in apparently healthy skin.

\section{Conflict of interest}

The authors declare no conflict of interest.

\section{References}

1. Nakamura T, Haider S, Colicino S, et al. Different definitions of atopic dermatitis: impact on prevalence estimates and associated risk factors. Br J Dermatol 2019; 181: 1272-9.

2. Heratizadeh A, Werfel T, Wollenberg A, et al. Effects of structured patient education in adults with atopic dermatitis: multicenter randomized controlled trial. J Allergy Clin Immunol 2017; 140: 845-53.

3. Williamson S, Merritt J, De Benedetto A. Atopic dermatitis in the elderly: a review of clinical and pathophysiological hallmarks. Br J Dermatol 2020; 182: 47-54.

4. Silverberg JI, Vakharia PP, Chopra R, et al. Phenotypical differences of childhood- and adult-onset atopic dermatitis. J Allergy Clin Immunol Pract 2018; 6: 1306-12.

5. Novak N, Leung DYM. Advances in atopic dermatitis. Curr Opin Immunol 2011; 23: 778-83.

6. Yang G, Seok JK, Kang HC, et al. Skin barrier abnormalities and immune dysfunction in atopic dermatitis. Int J Mol Sci 2020; 21: 2867.

7. Lee UH, Kim BE, Kim DJ, et al. Atopic dermatitis is associated with reduced corneodesmosin expression: role of cytokine modulation and effects on viral penetration. Br I Dermatol 2017; 176: 537-40.

8. Batista DIS, Perez L, Orfali RL, et al. Profile of skin barrier proteins (filaggrin, claudins 1 and 4) and Th1/Th2/Th17 cytokines in adults with atopic dermatitis. J Eur Acad Dermatol Venereol 2015; 29: 1091-5.

9. Halling-Overgaard AS, Kezic S, Jakasa I, et al. Skin absorption through atopic dermatitis skin: a systematic review. Br J Dermatol 2017; 177: 84-106.

10. Tsakok T, Woolf R, Smith CH, et al. Atopic dermatitis: the skin barrier and beyond. Br J Dermatol 2019; 180: 464-74.

11. Mu Z, Zhao Y, Liu X, et al. Molecular biology of atopic dermatitis. Clin Rev Allergy Immunol 2014; 47: 193-218.

12. Darlenski R, Kazandjieva J, Tsankov N. Skin barrier function: morphological basis and regulatory mechanisms. J Clin Med 2011; 4: 36-45. 
13. Menon GK, Cleary GW, Lane ME. The structure and function of the stratum corneum. Int J Pharm 2012; 435: 3-9.

14. Elias PM. Skin barrier function. Curr Allergy Asthma Rep 2008; 8: 299-305.

15. Arima K, Ohta S, Takagi A, et al. Periostin contributes to epidermal hyperplasia in psoriasis common to atopic dermatitis. Allergol Int 2015; 64: 41-8.

16. Liu Y, Shao Z, Shangguan G, et al. Biological properties and the role of IL-25 in disease pathogenesis. J Immunol Res 2018; 2018: 6519465.

17. Kouzaki H, Shimizu T. Interleukin-25 induces allergic inflammation. J Japan Soc Immunol Allergol Otolaryngol 2012; 30: 237-42.

18. Zhang X, Angkasekwinai P, Dong C, et al. Structure and function of interleukin-17 family cytokines. Protein Cell 2011; 2: 26-40.

19. Nakajima H, Tamachi T, Hirose K. Interleukin-25: a key initiator of type 2 immune responses. Cytokine Front Regulf Immune Respons Health Dis 2014; In: Cytokine Frontiers. Yoshimoto T, Yoshimoto T (eds.). Springer 2014; 247-66.

20. Valizadeh A, Khosravi A, Zadeh LJ, et al. Role of IL-25 in immunity. J Clin Diagn Res 2015; 9: OE01-4.

21. Suto H, Nambu A, Morita H, et al. IL-25 enhances Th17 cellmediated contact dermatitis by promoting IL-1 $\beta$ production by dermal dendritic cells. J Allergy Clin Immunol 2018; 142: 1500-9.

22. Brunner PM, Suárez-Farińas M, He H, et al. The atopic dermatitis blood signature is characterized by increases in inflammatory and cardiovascular risk proteins. Sci Rep 2017; 7: 8707.

23. Klonowska J, Glen J, Nowicki RJ, et al New cytokines in the pathogenesis of atopic dermatitis - new therapeutic targets. Int J Mol Sci 2018; 19: 3086.

24. Hvid M, Vestergaard C Kemp K, et al. IL-25 in atopic dermatitis: a possible link between inflammation and skin barrier dysfunction? I Invest Dermatol 2011; 131: 150-7.

25. Levin J, Friedlander SF, Del Rosso JQ. Atopic dermatitis and the stratum corneum - Part 1: the role of filaggrin in the stratum corneum barrier and atopic skin. J Clin Aesth Dermatol 2013; 6: 16-22.

26. Thyssen JP, Kezic S. Causes of epidermal filaggrin reduction and their role in the pathogenesis of atopic dermatitis. J Allergy Clin Immunol 2014; 134: 792-9.

27. Riethmuller C, McAleer MA, Koppes SA, et al. Filaggrin breakdown products determine corneocyte conformation in patients with atopic dermatitis. J Allergy Clin Immunol 2015; 136: $1573-80$

28. Drislane C, Irvine AD. The role of filaggrin in atopic dermatitis and allergic disease. Ann Allergy Asthma Immunol 2020; 124: 36-43.

29. Salimi M, Barlow JL, Saunders SP, et al. A role for IL-25 and IL-33-driven type-2 innate lymphoid cells in atopic dermatitis. J Exp Med 2013; 210: 2939-50.

30. Hänel KH, Cornelissen C, Lüscher B, et al. Cytokines and the skin barrier. Int J Mol Sci 2013; 14: 6720-45.

31. Horimukai K, Morita K, Narita M, et al. Transepidermal water loss measurement during infancy can predict the subsequent development of atopic dermatitis regardless of filaggrin mutations. Allergol Int 2016; 65: 103-8.

32. Guttman-Yassky E, Dhingra N, Leung DYM. New era of biologic therapeutics in atopic dermatitis. Exp Opin Biol Ther 2013; 13: 549-61
33. Guttman-Yassky E, Waldman A, Ahluwalia J, et al. Atopic dermatitis: pathogenesis. Semin Cutan Med Surg 2017; 36: 100-3.

34. Aktar MK, Kido-Nakahara M, Furue M, et al. Mutual upregulation of endothelin-1 and IL-25 in atopic dermatitis. Allergy Eur J Allergy Clin Immunol 2015; 70: 846-54.

35. Corrigan CJ, Wang W, Meng Q, et al. Allergen-induced expression of IL-25 and IL-25 receptor in atopic asthmatic airways and late-phase cutaneous responses. J Allergy Clin Immunol 2011; 128: 116-24.

36. Roan F, Obata-Ninomiya K, Ziegler SF. Epithelial cell-derived cytokines: more than just signaling the alarm. J Clin Investig 2019; 129: 1441-51.

37. Aalberse JA, van Thuijl AO, Meijer Y, et al. Plasma IL-25 is elevated in a subgroup of patients with clinical reactivity to peanut. Clin Transl Allergy 2013; 3: 40.

38. Martel BC, Dyring-Andersen B, Skov L, et al. Different cytokine profiles of skin-derived $T$ cell cultures from patients with atopic dermatitis and psoriasis. Inflamm Res 2016; 65: 265-72.

39. Nakamura N, Tamagawa-Mineoka R, Maruyama A, et al. Stratum corneum interleukin-25 expressions correlate with the degree of dry skin and acute lesions in atopic dermatitis. Allergol Int 2020; 69: 462-4.

40. Lacy P, Stow JL. Cytokine release from innate immune cells: association with diverse membrane trafficking pathways. Blood 2011; 118: 9-18

41. Ungar B, Garcet S, Gonzalez J, et al. An integrated model of atopic dermatitis biomarkers highlights the systemic nature of the disease. I Invest Dermatol 2017; 137: 603-13.

42. Hamilton JD, Suárez-Farińas M, Dhingra N, et al. Dupilumab improves the molecular signature in skin of patients with moderate-to-severe atopic dermatitis. J Allergy Clin Immunol 2014; 134: 1293-300.

43. De Benedetto A, Yoshida T, Fridy S. Histamine and skin barrier: are histamine antagonists useful for the prevention or treatment of atopic dermatitis? J Clin Med 2015; 4: 741-55.

44. Suárez-Farińas M, Tintle SJ, Shemer A, et al. Nonlesional atopic dermatitis skin is characterized by broad terminal differentiation defects and variable immune abnormalities. J Allergy Clin Immunol 2011; 127: 954-64.

45. De Koning HD, Kamsteeg M, Rodijk-Olthuis D, et al. Epidermal expression of host response genes upon skin barrier disruption in normal skin and uninvolved skin of psoriasis and atopic dermatitis patients. J Investig Dermatol 2011; 131: 263-6.

46. Polańska A, Dańczak-Pazdrowska A, Silny W, et al. Nonlesional skin in atopic dermatitis is seemingly healthy skinobservations using noninvasive methods. Videosurgery Miniinv 2013; 8: 192-9.

47. Thijs JL, Strickland I, Bruijnzeel-Koomen CAFM, et al. Moving toward endotypes in atopic dermatitis: identification of patient clusters based on serum biomarker analysis. J Allergy Clin Immunol 2017; 140: 730-7. 